\title{
Merging Analytic and Empirical GEO Debris Synchronization Dynamics
}

\author{
Paul V. Anderson ${ }^{1}$ \\ University of Colorado, Boulder, USA \\ Darren S. McKnight and Frank Di Pentino \\ Integrity Applications Incorporated, USA \\ Hanspeter Schaub ${ }^{2, *}$ \\ University of Colorado, Boulder, USA
}

\begin{abstract}
The motion of abandoned satellites near the geostationary (GEO) region has been extensively studied, modeled, and compared to the motion of stationkept, operational satellites, providing insights into the evolution of uncontrolled orbits at GEO. Analytic developments produced a family of curves represented in the ascending node versus inclination space describing the long-term precession of the orbit plane at GEO, and forecasted the clustering of objects at the geopotential wells. However, recent investigations were undertaken to characterize apparent anomalistic behavior of GEO objects and classification of objects into related families. This paper provides a unifying summary of early bottom-up analytical theory with more recent top-down operational observations, highlighting the common linkage between these dimensions of GEO object behavior. This paper also identifies the relevance of these patterns of life tendencies for future operations at and near
\end{abstract}

\footnotetext{
* Corresponding author

Email addresses: paul.anderson@colorado.edu (Paul V. Anderson), dmcknight@integrity-apps.com, fdipentino@integrity-apps.com (Darren S. McKnight and Frank Di Pentino), Hanspeter.Schaub@colorado.edu (Hanspeter Schaub)

${ }^{1}$ Graduate Research Assistant

${ }^{2}$ Professor, Associate Chair, Alfred T. and Betty E. Look Professor of Engineering
}

Preprint submitted to Advances in Space Research

April 9, 2016

(C) 2016. This manuscript version is made available under the Elsevier user license http://www.elsevier.com/open-access/userlicense/1.0/ 
GEO, and discusses the long-term implications of these patterns of life for space situational awareness activities in this regime.

Keywords: Space Debris, Geosynchronous Ring, Conga Line Motion

\section{Introduction}

Visualizations of the geosynchronous (GEO) debris population from the longitude slot-relative perspective of the Earth-centered, Earth-fixed frame demonstrate that the collective, once-daily motion of this population is similar to a transverse wave circulating around the GEO ring with a period of one sidereal day. (McKnight and Di Pentino, 2013) Thus, daily latitude cycles for uncontrolled objects neighboring one another in longitude are not, in general, out-of-phase. Figure 1 shows the longitude/latitude distribution of the GEO debris population extracted from the 28 February 2014 two-line element (TLE) set, at four different times during 01 March 2014, to illustrate this systematic correlation in latitude. As time advances, the peak and trough of this transverse "debris wave" shift westward linearly in longitude. As will be shown in this paper, apparent latitudinal synchronization of the GEO debris population is driven by a combination of inclination and clustering in right ascension of the ascending node (RAAN), the latter resulting from operators leveraging naturally-occurring luni-solar perturbations to reduce north-south station-keeping effort. (Capelle and Sharma, 2000)

Upon close inspection of Figure 1 (and especially if animating the motion of Figure $1^{3}$ ), eight objects in the population qualitatively appear to be either fully or partially unsynchronized with the wave-like latitudinal motion exhibited by the rest of the population. When the local debris populations at or near the longitudes of these outliers are rising in latitude from south to north through the equatorial plane, these objects are descending in latitude from north to south, against the flow of the predictable "patterns of life" nominally observed for derelict motion at GEO. This discrepancy in the latitude cycle indicates that the descending nodes of the outliers are currently located near the ascending nodes of the synchronized objects clustered in neighboring longitude slots. This paper is thereby borne out of the quest to characterize the apparent anomalous motion of these eight outlying objects,

\footnotetext{
${ }^{3}$ See animation of daily latitudinal motion of derelict GEO population at http:// hanspeterschaub.info/Movies/GeoDebrisConga.mp4.
} 

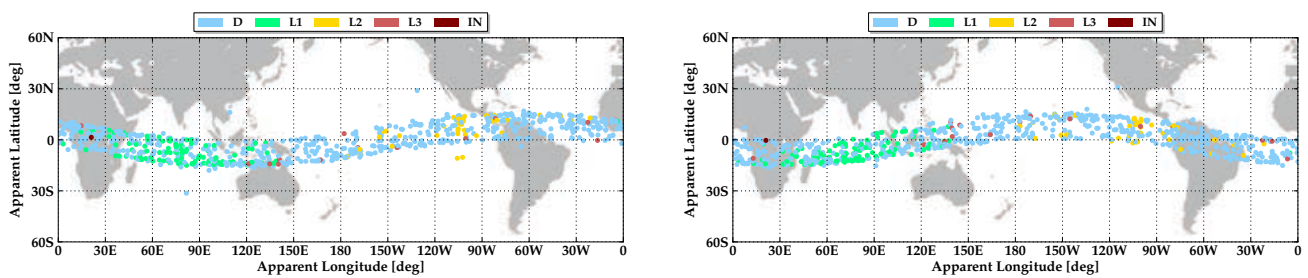

(a) Longitude/latitude distribution of de- (b) Longitude/latitude distribution of debris population (00:00:00 Zulu). bris population (06:00:00 Zulu).
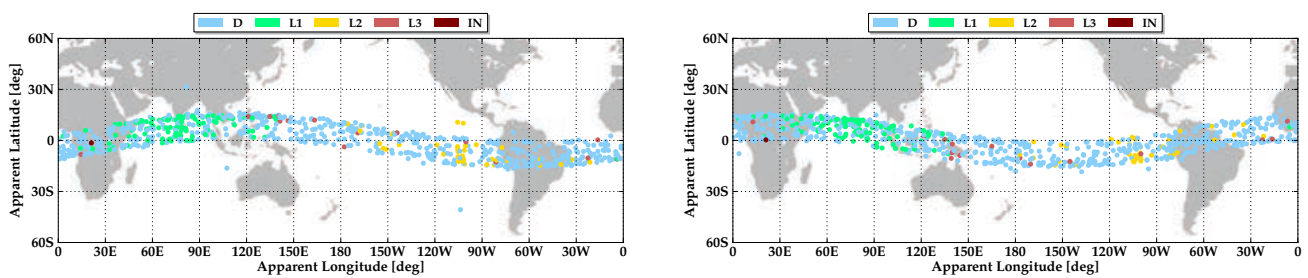

(c) Longitude/latitude distribution of debris (d) Longitude/latitude distribution of depopulation (12:00:00 Zulu).

bris population (18:00:00 Zulu).

Figure 1: Longitude/latitude distribution of the GEO debris population on 01 March 2014, shown in six-hour intervals to illustrate the collective, wave-like behavior of the 745 derelicts comprising this debris population. Objects are colored by uncontrolled orbit class, as listed in Issue 16 of the Classification of Geosynchronous Objects report maintained by the European Space Agency. (Flohrer, 2014)

by studying whether such asynchronicity in latitude can arise under wellknown RAAN dynamics at GEO driven by the coupling between luni-solar perturbations and central body oblateness. (Chao, 2005)

Specifically, this paper provides a unifying summary of "bottom-up" analytical theory with "top-down" observational data to highlight the common linkage between these two dimensions of GEO debris behavior. The relevance of naturally-occurring patterns of debris motion at GEO to current and future operations in the GEO arena is discussed, and long-term implications of these tendencies to space situational awareness (SSA) in this arena are highlighted. 


\section{Inclination and RAAN Variations Induced by Luni-Solar Per- turbations}

The doubly-averaged ${ }^{4}$ differential equations of motion governing inclination $i$ and right ascension of the ascending node $\Omega$ variations induced by luni-solar perturbations prominent at the GEO altitude are, for near-circular orbits only, given by (Chao, 2005; Chobotov, 2002)

$$
\begin{aligned}
& \frac{\mathrm{d} i}{\mathrm{~d} t}=\sum_{j=1}^{2} \frac{3}{8} \gamma_{j}\left[\cos (i) \sin \left(2 i_{j}\right) \sin \left(\Omega-\Omega_{j}\right)\right. \\
&\left.+\sin (i) \sin ^{2}\left(i_{j}\right) \sin \left(2\left(\Omega-\Omega_{j}\right)\right)\right] \\
& \frac{\mathrm{d} \Omega}{\mathrm{d} t}=-\frac{3}{2} J_{2}\left(\frac{R_{\oplus}}{a}\right)^{2} n \cos (i) \\
&+\sum_{j=1}^{2} \frac{3}{16}\left(\frac{\gamma_{j}}{\sin (i)}\right)\left[\sin (2 i)\left(1-3 \cos ^{2}\left(i_{j}\right)\right)\right. \\
& \quad+2 \cos (2 i) \sin \left(2 i_{j}\right) \cos \left(\Omega-\Omega_{j}\right) \\
&\left.\quad+\sin (2 i) \sin ^{2}\left(i_{j}\right) \cos \left(2\left(\Omega-\Omega_{j}\right)\right)\right]
\end{aligned}
$$

where the summations are performed over the Sun and the Moon, respectively, with $\gamma_{j} \equiv n_{j}^{2} R_{m} / n$ for third-body mass ratios $R_{m}=1$ for the Sun and $R_{m}=1 / 82.3$ for the Moon. (Chobotov, 2002) In this formulation, $i, \Omega, n$, and $a$ denote the inclination, RAAN, mean motion, and semi-major axis of the uncontrolled derelict, respectively, while $i_{j}, \Omega_{j}$, and $n_{j}$ denote third-body inclination with respect to the equatorial plane, third-body RAAN with respect to the line of Aries, and third-body mean motion (i.e., approximately one revolution per year for the Sun and one revolution per sidereal month for the Moon). Note that the coupling between luni-solar perturbations and central body oblateness is incorporated in Equation (2) via the well-known term that describes secular regression in $\Omega$ induced by the $J_{2}$ zonal harmonic. (Vallado, 2007)

\footnotetext{
${ }^{4}$ Termed "doubly" averaged because the complete equations of motion for inclination and RAAN have been averaged once over true anomaly, and a second time over argument of perigee, such that short-period and long-period oscillations in inclination and RAAN have been removed, leaving secular variations only. (Chao, 2005)
} 
Equations (1)-(2) offer insight into the long-term evolution of uncontrolled orbits in the GEO regime. In particular, Equation (1) indicates that inclination drift will be zero inasmuch as $\Omega-\Omega_{j}=0^{\circ}$ or $\Omega-\Omega_{j}=180^{\circ}$, i.e., the line of nodes for the uncontrolled object is aligned with that of the third body. (Chao, 2005) Without routine north-south station-keeping maneuvers, Equations (1)-(2) combine to drive a long-term, cyclical precession of the orbit plane, in which correlated motions of RAAN and inclination over an approximate 53-year period are analogous to the precession and nutation of a gyroscope. (Capelle and Sharma, 2000) Physically, out-of-plane force components acting on the uncontrolled object torque the orbit plane along the line of nodes, resulting in the angular momentum vector precessing about an intermediate axis, the unit vector normal to the Laplace plane, which is displaced approximately $7.4^{\circ}$ from Earth's polar rotation axis towards the ecliptic pole. (Allan and Cook, 1964; Friesen et al., 1993)

Long-term, coupled inclination and RAAN variations described by Equations (1)-(2) are visualized using the well-known phase portrait in Figure 2(a), which shows the clockwise trajectories that uncontrolled objects traverse in this inclination and RAAN phase space. Objects beginning with low inclination and a RAAN within Quadrants I or IV will experience a periodic, egg- or triangular-shaped (Type I) progression within this phase space, while objects initialized with a RAAN in Quadrants II and III experience a periodic, bell-shaped (Type II) progression. (Capelle and Sharma, 2000) For Type I motion, the node advances and regresses through the minimum and maximum inclinations of the cycle, respectively, each occurring at $\Omega=0^{\circ}$. For Type II motion, the node continually regresses from minimum to maximum inclinations and back - nodal advancement is not experienced along these bell-shaped, Type II progressions. In particular, Reference Zhao et al. (2015) provides analytical expressions for the predicted variation range of inclination as a function of initial inclination and RAAN in the phase plane:

$$
\Delta i= \begin{cases}{\left[i_{*}-2 \alpha, i_{*}\right]:} & i_{*} \geqslant 2 \alpha \\ {\left[2 \alpha-i_{*}, i_{*}\right]:} & \alpha \leqslant i_{*} \leqslant 2 \alpha \\ {\left[i_{*}, 2 \alpha-i_{*}\right]:} & i_{*} \leqslant \alpha\end{cases}
$$

where $i_{*}$ denotes maximum or minimum inclination when $\Omega=0^{\circ}$, and $\alpha \approx 7.4^{\circ}$ is the inclination of the intermediate Laplace plane with respect to Earth's equatorial plane.

The red line in Figure 2(a) highlights the "separatrix-like," triangularshaped boundary between Type I/II progressions, (Vaughan and Mullikin, 


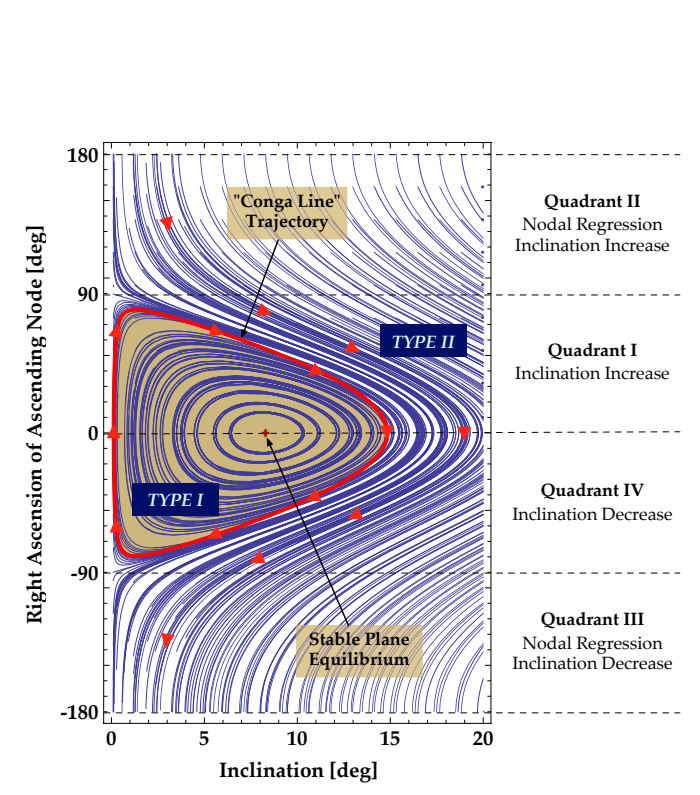

(a) Phase portrait visualization of inclination and RAAN variations.

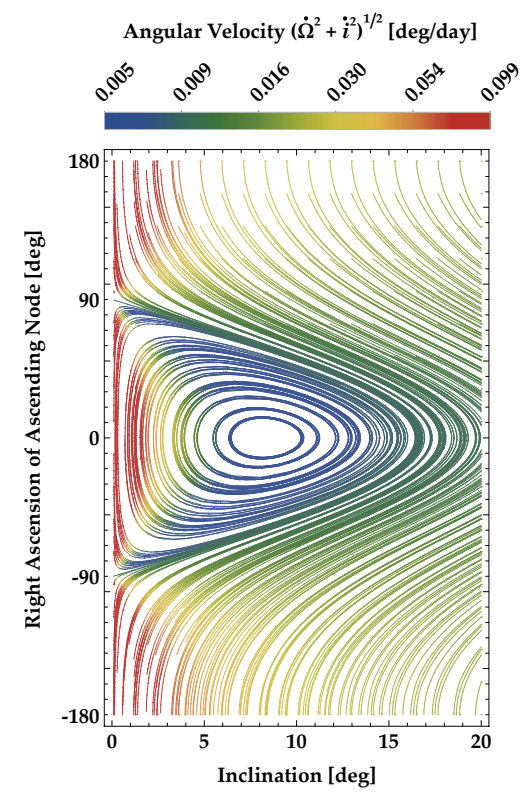

(b) Angular rates on phase portrait trajectories.

Figure 2: Phase portraits illustrating admissible trajectories in inclination and RAAN phase space, governed by the doubly-averaged Equations (1)-(2) for inclination and RAAN variations driven by luni-solar perturbations at the GEO altitude.

1995) informally termed the "conga-line" trajectory ${ }^{5}$ because derelict GEO objects oscillate north-south along the line of apsides relative to operational assets at GEO. GEO operators reduce north-south station-keeping fuel allocation by initializing their satellites with several degrees in inclination and RAAN in Quadrant IV, such that over the nominal lifespan of these satellites, luni-solar perturbations naturally decrease the inclination to nearly zero before the 53-year cycle begins increasing inclination at a rate of approximately $0.8^{\circ}$ per year. (Yasaka et al., 1999) If desired, routine station-keeping maneuvers can be executed to re-initiate this portion of the triangularshaped progression when inclination begins to exceed the operational requirement. (Capelle and Sharma, 2000) As a result, since the conga-line progression in Figure 2(a) best leverages natural perturbations to minimize

\footnotetext{
${ }^{5}$ See McKnight and Di Pentino (2013) and McKnight et al. (2013).
} 
expensive inclination control burns, which comprise a significant portion of station-keeping fuel budgets, (Chao, 2005) maintaining a naturally-adjusted inclination has been historically popular among GEO operators.

Figure 2(a) also illustrates the existence of the well-known GEO stable plane, which is a fixed point $(\dot{i}=\dot{\Omega}=0)$ of the doubly-averaged Equations (1)-(2). Objects initialized at this equilibrium configuration with $i_{0} \approx 7.4^{\circ}$ and $\Omega_{0} \approx 0^{\circ}$ exhibit dramatically-reduced inclination and RAAN variations over the 53-year cycle. Inclination variations are confined to within $1.2^{\circ}$ of the initial plane in this setup, in comparison to the $12-15^{\circ}$ excursions typical for Type I and II progressions in Figure 2(a). (Friesen et al., 1993) Physically, the stable plane equilibrium results from luni-solar perturbations acting against the nodal regression driven by central body $J_{2}$ oblateness, such that these equal-but-opposite effects negate each other. The GEO stable plane has important operational implications for satellite collocation, conjunction velocities, and GEO mitigation alternatives. (Friesen et al., 1993)

Since both Type I and Type II cycles in Figure 2(a) have a period of approximately 53 years - even though inclination and RAAN variations are, in general, smaller for Type I cycles than for Type II cycles-Figure 2(b) shows

the non-uniformity of the angular speed $\sqrt{\dot{\Omega}^{2}+\dot{i}^{2}}$ along these progressions. As is predicted analytically with Equation (2), rapid nodal advancement occurs at small inclinations, when the luni-solar contributions to Equation (2) dominate $J_{2}$-induced nodal regression. (Vaughan and Mullikin, 1995) Type II motion exhibits a higher angular rate than "inner" Type I motion, such that each cycle maintains the 53-year period. Thus, although smart orbital insertion can leverage the naturally-occurring inclination management to reduce the orbit from several degrees of inclination to equatorial, this is the most rapid part of the progression (lasting about three years), requiring re-initiation every time the inclination begins exceeding operational limits (e.g., five times during a nominal 15-year operational lifespan). (Capelle and Sharma, 2000)

\section{Current Inclination and RAAN Distribution}

In light of the bottom-up, analytic theory presented in Section 2, it is instructive to consider the distribution of both controlled GEO satellites and uncontrolled GEO derelicts in the inclination/RAAN phase portrait in Figure 2. GEO objects are extracted from publicly-available TLE sets according 
to eccentricity less than 0.2 , inclination less than $70^{\circ}$, and mean motion 0.9 1.1 revs per sidereal day. (Flohrer, 2014) The 1145 GEO objects extracted from the 28 February 2014 TLE set are classified using the taxonomy maintained by ESA in its "Classification of Geosynchronous Objects" reports. Table 1 describes this GEO classification system.

Table 1: Orbit classifications for categorizing GEO objects. (Flohrer, 2014; Anderson and Schaub, 2013)

\begin{tabular}{c|l} 
Class & \multicolumn{1}{|c}{ Class Description } \\
\hline C1 & Longitude (E-W) and inclination (N-S) control \\
C2 & Longitude control only (E-W control only) \\
D & Circulating above/below/through GEO ring \\
L1 & Libration about Eastern gravity well at $75^{\circ} \mathrm{E}$ \\
L2 & Libration about Western gravity well at $105^{\circ} \mathrm{W}$ \\
L3 & Libration about both gravity wells \\
IN & Unknown status (i.e., recent TLE not available)
\end{tabular}

Figure 3 provides the phase portrait distribution for the 400 controlled GEO satellites from this population, 318 of which are class C1, and 82 of which are class C2. (Flohrer, 2014) In particular, Figure 3(a) shows this controlled distribution colored by orbit class. The majority of class $\mathrm{C} 1$ satellites are maintaining near-zero inclination by either (a) executing routine station-keeping burns, or (b) leveraging the conga-line trajectory or a variation thereof for a naturally-occurring inclination decrease, as described in Section 2. Conversely, the majority of C2 satellites - those that (a) were not equipped to perform out-of-plane station-keeping, (b) have this capability but have operators who choose not to exercise it (for reasons including relocation, change in ownership, change in mission, etc.), or (c) have lost this capability on-orbit - are progressing on or near the intermediate conga-line trajectory. ${ }^{6}$ Figure 3(b), which illustrates this controlled distribution colored by launch year, confirms that class $\mathrm{C} 2$ satellites furthest along the clockwise, 53-year cycle (i.e., have largest inclinations in Quadrant I) are the oldest objects in the operational population. As a result, given two collocated $\mathrm{C} 2$ objects in this synchronized configuration, the younger object will lag behind the older

\footnotetext{
${ }^{6}$ Recall that the conga-line trajectory and the doubly-averaged Equations (1)-(2) provide approximate, analytic, first-order descriptions of actual inclination and RAAN variations experienced at the GEO altitude.
} 


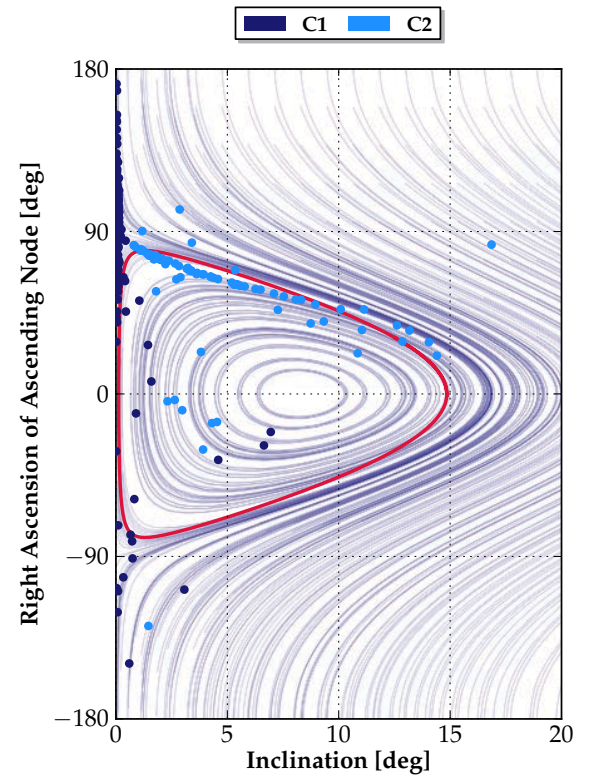

(a) Distribution of controlled $\mathrm{C} 1 / \mathrm{C} 2$ satellites.

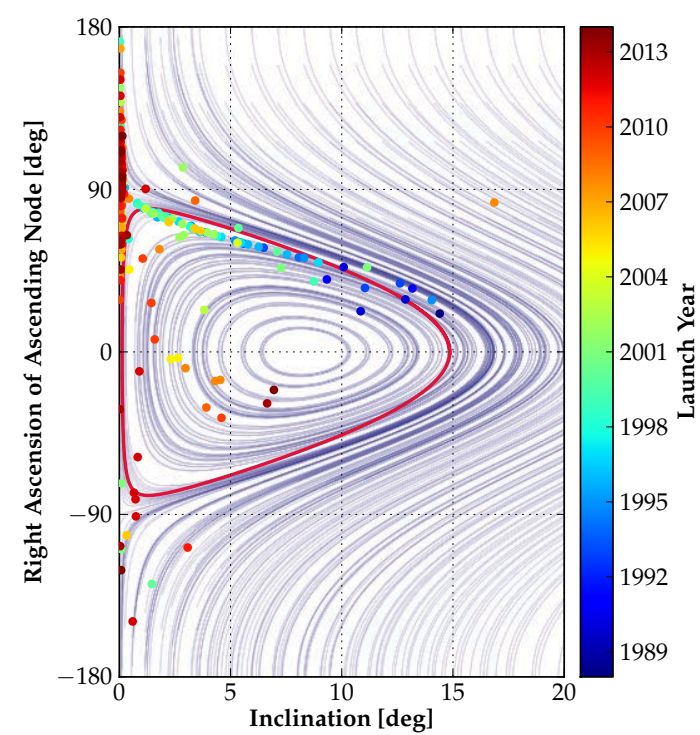

(b) Controlled distribution colored by launch year.

Figure 3: Phase space distribution of controlled GEO satellites on 28 February 2014.

object in the once-daily latitude cycle, because the node of the younger object has not regressed as far as that of the older object. ${ }^{7}$ Ultimately, the dichotomy between fully-controlled $\mathrm{C} 1$ satellites and partially-controlled $\mathrm{C} 2$ satellites in Figure 3 demonstrates that while out-of-plane station-keeping is used to maintain near-equatorial orbits for C1 satellites, coupled inclination and RAAN variations exhibited by $\mathrm{C} 2$ objects follow predictable progressions described by Equations (1)-(2).

Figure 4 provides the phase space distribution for the 745 uncontrolled GEO objects from the 28 February 2014 TLE set, which are classified into one drift class (D), three librating classes (L1/L2/L3), and one indeterminate class (IN). (Flohrer, 2014) Prominent clustering in derelict objects is observed on or near the conga-line trajectory, especially around the apex of this triangular-shaped progression, where the inclination achieves a maxi-

\footnotetext{
${ }^{7}$ Latitudinal lag between collocated satellites of differing on-orbit age has been confirmed in animations and numerical simulations performed by the co-authors at Integrity Applications, Inc.
} 

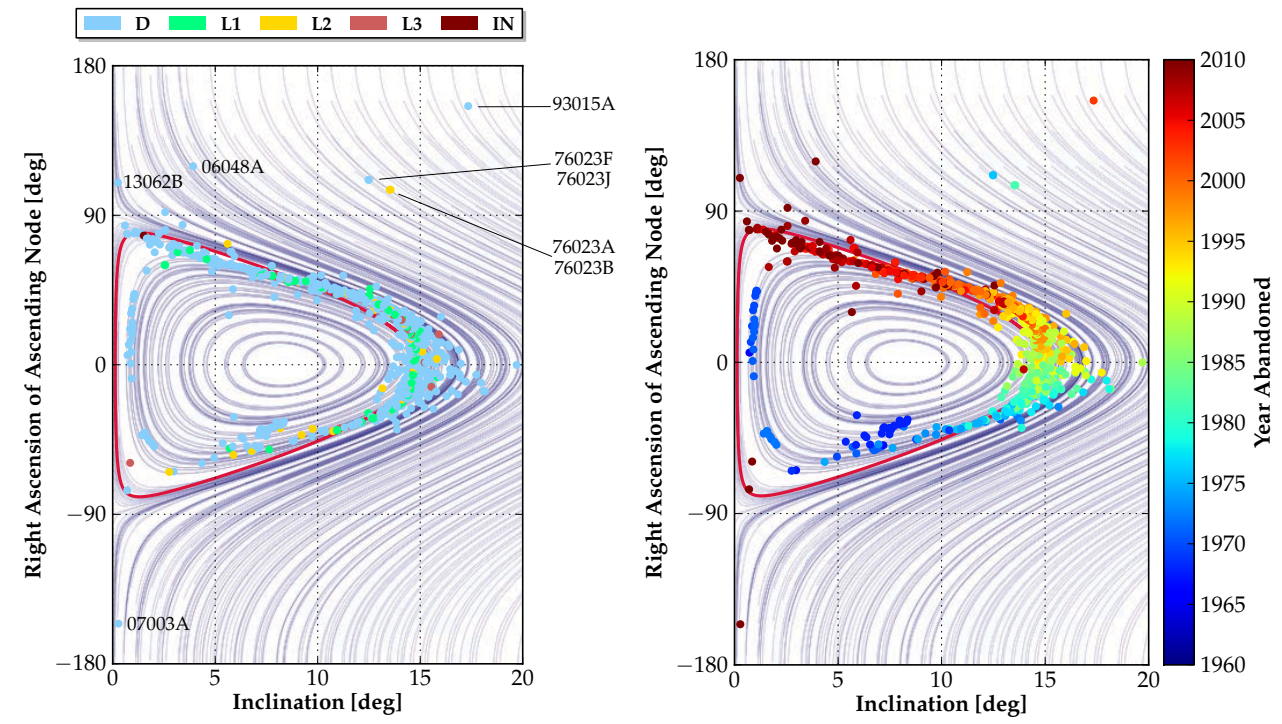

(a) Distribution of uncontrolled (b) Uncontrolled distribution colored by D/L1/L2/L3/IN objects. year abandoned.

Figure 4: Phase space distribution of uncontrolled GEO objects on 01 March 2014.

mum value of approximately $15^{\circ}$ over the 53 -year cycle. Sharma et al. (2002) shows that in 2002 (39 elapsed years since the first GEO utilization in 1963), the majority of uncontrolled objects at GEO had not yet crossed the apex of this systematic progression. Now, 13 years later, Figure 4 illustrates that a significant portion of this uncontrolled population has already crossed, or is currently crossing, into the second half of the conga line cycle (from Quadrant I to Quadrant IV), in which the RAAN continues to regress while the inclination begins decreasing. Since 52 years have now elapsed since the first GEO utilization, the first GEO debris objects generated in the 1960sincluding the world's first GEO communications satellite, Syncom 2-are soon to complete a full Type I revolution in inclination and RAAN phase space.

Figure 4(a), which shows the uncontrolled debris distribution colored by orbit class, demonstrates that factors such as longitude of abandonment or orbit class - whether drifting or librating about one or both of the gravitational wells - do not impact the phase space progression that is followed by a debris 
object. ${ }^{8}$ It is important to emphasize that inclination and RAAN at the moment the object is abandoned dictate whether a Type I or Type II progression will result in the following cycle. Since luni-solar gravitational perturbations are conservative, the resulting cycles governed by Equations (1)-(2) are periodic, and do not admit natural transition from Type I to Type II behavior, or vice-versa, under luni-solar perturbations alone. The apparent outlying objects labeled in Figure 4(a), discussed in Section 5, could result from one or more of the following possibilities or artificial means:

1. When operational, these satellites were not station-kept to minimum inclination management - or to inner, Type I motion - for mission-related reasons, or (for launch debris) were not initially inserted onto Type I cycles.

2. End-of-life disposal was attempted, and the maneuver sequence, whether purposefully or inadvertently, altered the inclination/RAAN while raising the semi-major axis.

3. Since Gauss's variational equations show that any out-of-plane acceleration will simultaneously change both inclination and RAAN, (Schaub and Junkins, 2009) an out-of-plane collision with either environmental or artificial debris occurred, or the object has vented (or is currently venting) propellant with an out-of-plane component.

Although these possibilities are not exhaustive in scope, they enforce the fact that initial conditions in inclination and RAAN phase space dictate whether a Type I or II cycle will result. Under the doubly-averaged Equations (1)-(2), the Type I and II progressions are mutually exclusive.

To illustrate how age on-orbit can be approximated by the phase portrait of Figure 2, Figure 4(b) illustrates the uncontrolled object distribution colored by estimated year abandoned. For upper stages and other launch or mission-related debris (e.g., apogee kick motors), the year of abandonment is the launch year; for derelict payloads in the population, the year of abandonment is approximated using a linear lifespan model derived from historical

\footnotetext{
${ }^{8}$ Although orbit class does not affect the progression directly, the semi-major axis (which is one of the critical factors in determining whether an object will drift or librate once uncontrolled (Allan, 1963)) is a parameter of Equation (2), and is assumed to be constant when propagating this differential equation. This is a valid assumption given the magnitude of naturally-occurring semi-major axis variations at GEO, which are insignificant relative to the $42164 \mathrm{~km}$ radius of the GEO ring (e.g., $60^{\circ}$ of libration amplitude results from approximately $\pm 30 \mathrm{~km}$ of semi-major axis variation (Zhao et al., 2013)).
} 
lifespan data, which reflects a linear increase from six years on average in 1977 to 13 years on average in 2010. (McKnight and Di Pentino, 2013) Similar to Figure 3(b), Figure 4(b) demonstrates that derelicts further along the triangular-shaped progression are older than those near the "beginning" of the progression (i.e., near $i \approx 0^{\circ}$ and $\Omega \approx 90^{\circ}$ ). GEO debris abandoned in the late 1980s have achieved the maximum inclination of the cycle at the apex of the progression, while derelicts abandoned in the 1960s (or, approximately 26-27 years earlier) are now moving through the minimum inclination of the cycle towards one full revolution in the phase space. Assuming that a particular payload of interest - operational or defunct - was station-kept, the age distribution of Figure 4(b) can be leveraged with the angular rate information of Figure 2(b) to estimate either when this object was abandoned, or when it lost its north-south station-keeping capability (if currently operational).

\section{Evolution of Inclination and RAAN Distribution}

In addition to studying the present-day distribution of GEO debris objects in inclination and RAAN space, it is beneficial to forecast the evolution of this distribution under the doubly-averaged Equations (1)-(2). Figure 5 illustrates the approximate distributions in a predicted 20, 40, and 60 years, as compared to the phase space distribution on 01 March 2014, shown in Figure 4(a). As noted in Section 3, a significant proportion of the present-day debris population is crossing into the second half of the conga-line progression, giving rise to strong clustering in RAAN about the line of Aries $\left(\Omega=0^{\circ}\right)$. Systematic, predictable clustering in RAAN has been widely leveraged for space-based space surveillance (SBSS), sensor tasking, and space situational awareness activities at GEO. (Sharma et al., 2002) By pointing ground- or space-based sensors towards the well-known GEO "pinch points," observational coverage of the resident space object (RSO) population at GEO can be maximized, and the probability of detecting "new" objects for TLE catalog maintenance is improved. The productivity of the Space-Based Visible sensor on the Midcourse Space Experiment satellite improved $20-30 \%$ by leveraging these natural GEO pinch points. (Capelle and Sharma, 2000)

However, as Figure 5 illustrates, the interplay between location in the phase space and the location-dependent angular rates in Figure 2(b) gives rise to the periodic dispersion and eventual re-focusing in the RAAN distri-

bution. As Type I derelicts circulate within the phase space, the spread of this distribution expands from reflecting Quadrant I clustering over $\left[0^{\circ}, 90^{\circ}\right]$ 

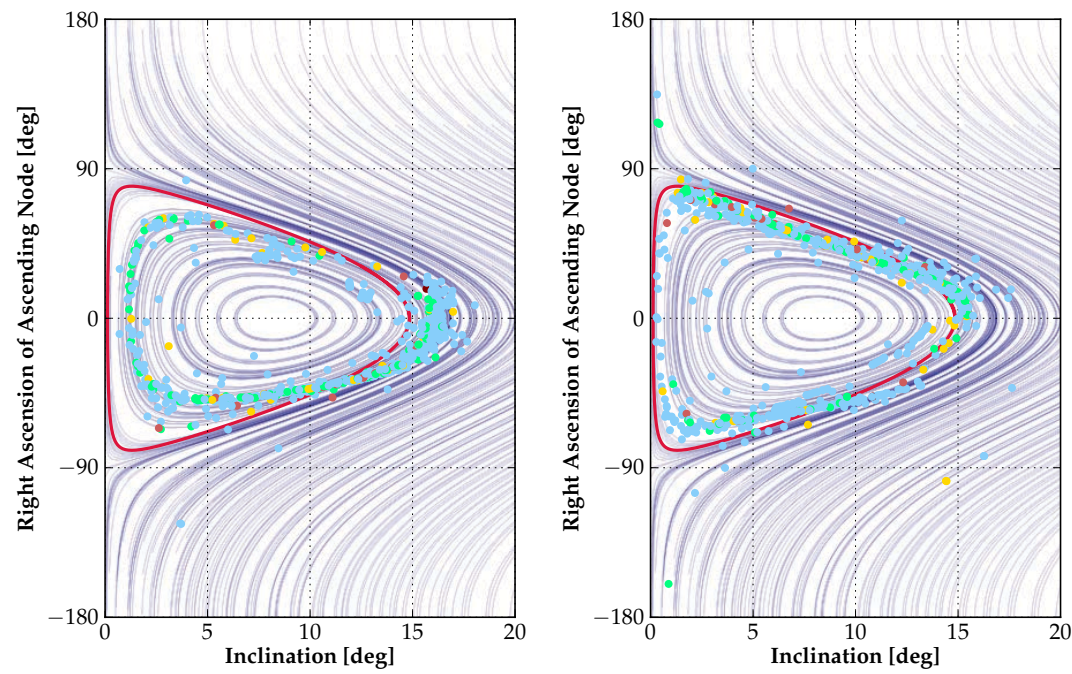

(a) Predicted distribution in 20 (b) Predicted distribution in 40 years. years.

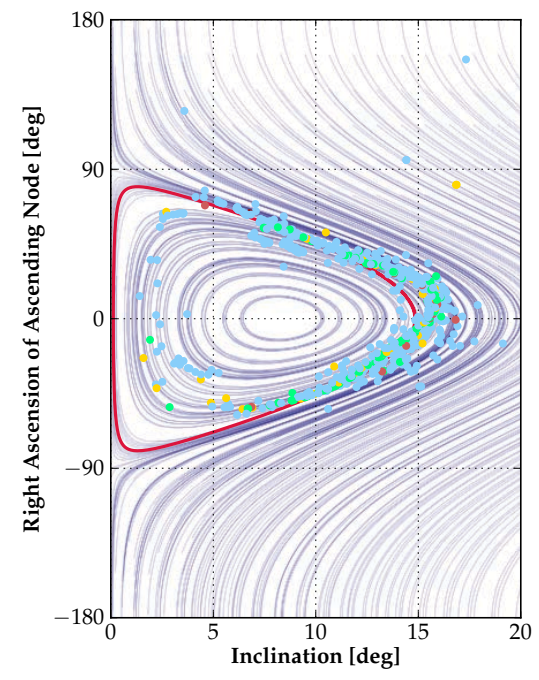

(c) Predicted distribution in 60 years.

Figure 5: Evolution of derelict motion at GEO in inclination/RAAN space over 60 years, predicted using the doubly-averaged Equations (1)-(2) for secular inclination and RAAN variations driven by luni-solar perturbations at the GEO altitude. 
Table 2: Eight GEO debris objects exhibiting unsynchronized latitude cycles as of 01 March 2014.

\begin{tabular}{l|c|c}
\multicolumn{1}{c|}{ Common Name } & COSPAR & Class \\
\hline LES-8 & $76023 \mathrm{~A}$ & L2 \\
LES-9 & $76023 \mathrm{~B}$ & L2 \\
Titan 3C Transtage R/B & $76023 \mathrm{~F}$ & $\mathrm{D}$ \\
LES Operational Debris & $76023 \mathrm{~J}$ & $\mathrm{D}$ \\
USA 98 (UFO F1) & $93015 \mathrm{~A}$ & $\mathrm{D}$ \\
Sinosat-2 (Xinnuo-2) & 06048A & D \\
BeiDou-1D (BeiDou-4) & 07003A & D \\
Proton-M Briz-M R/B & $13062 \mathrm{~B}$ & $\mathrm{D}$
\end{tabular}

to reflecting full, Quadrant I/IV clustering over $\left[-90^{\circ}, 90^{\circ}\right]$. The implication of this dispersion of objects to SSA is that the GEO pinch points are spreading out, thus diminishing in usefulness for debris observation campaigns that employ ground or space-based sensors to survey these particular right ascensions in the equatorial plane. In order to establish a leak-proof fence without extensive declination stripe scanning at the pinch points, a more substantial search region over right ascension must be surveyed to maintain productivity levels met by previous observation campaigns. (Schildknecht, 2007) ${ }^{9}$ Beneficially, Figure 5 shows that dispersion in RAAN does not continue indefinitely; rather, the distribution begins to re-focus as the majority of the population passes through the minimum-inclination portion of the 53-year cycle to complete one Type I revolution, after which nodal regression rates slow. The spread in this RAAN distribution thus expands and contracts as luni-solar perturbations and Earth's oblateness propel the derelict population around the conga line cycle.

\section{Investigating Unsynchronized Objects}

In light of the analytic theory presented in Section 2, and the observational portrait of the current inclination and RAAN distribution in Section 3, the eight derelicts identified as being unsynchronized with the transverse, wave-

\footnotetext{
${ }^{9}$ Leak-proof search patterns for the entire GEO regime beyond conventional pinch point surveys are presented for ground-based and space-based architectures in (Flohrer et al., 2005) and (Flohrer et al., 2011), respectively.
} 
like behavior shown in Figure 1 are now examined. Animations of the daily latitude cycle of the GEO derelict population indicate that these "outliers" traverse latitude cycles either partially or fully out-of-phase with those exhibited by nearby derelicts in neighboring longitude slots. As noted earlier, two latitude cycles become more out-of-phase the farther the corresponding RAAN angles are displaced from one another, e.g., two objects with RAAN angles that are $180^{\circ}$ apart will be completely unsynchronized in the latitude cycle. Two asynchronous latitude cycles are best visualized in right ascension and declination coordinates, in which these daily cycles appear as out-of-phase sine waves instead of "figure-8" analemmas. (Capelle and Sharma, 2000; Sharma et al., 2002)

For purposes of flight safety and on-orbit anomaly correlation, it is important to understand why the RAAN angles for these eight outliers - provided in Table 2-are not clustered with those of the rest of the GEO debris population from the 28 February 2014 TLE set. The equatorial crossing windows for the synchronized debris population are episodic, predictable, and dependent on time of day in addition to time of year. These alternating, six-hour intervals of increasing and decreasing flux relative to any given longitude slot are important for risk assessment and prediction at GEO, since conjunction events are most likely to occur in the out-of-plane direction when the longitude slot is passing through either the ascending or descending node pinch point. Derelicts that are unsynchronized with this "pattern of life" present a special hazard in that these objects are crossing the equatorial plane when north-south flux levels are at the daily minimum. ${ }^{10}$

As the current inclination and RAAN distribution in Figure 4 shows, the eight unsynchronized derelicts in Table 2 are currently positioned in Quadrants II and III, away from the significant RAAN clustering occurring at the boundary between Quadrants I and IV. These objects are not exhibiting oscillatory Type I trajectories in the phase space; rather, they are following bell-shaped Type II progressions, according to Equations (1)-(2). In contrast to the Type I cycles, which exhibit long durations of nodal regression followed by shorter periods of nodal advancement near the minimum inclination of the cycle, Type II cycles exhibit continual nodal regression over the entirety of

\footnotetext{
${ }^{10}$ As an analogy, consider the hazard to one's vehicle when driving on the highway. Although risk levels during rush hour can be reasonably characterized in that they spike during predictable time frames, the risk attributed to a rogue driver speeding southbound down the northbound side is challenging to characterize.
} 
the 53-year cycle. This critical difference is illustrated in Figure 6, which provides the predicted RAAN trajectories over a 60 -year time frame for various synchronized and unsynchronized objects in the 28 February 2014 TLE set. Figure 6(a) provides the RAAN trajectories for eight initially synchronized objects, that is, with RAAN angles within one standard deviation of the distribution mean. The RAAN angles for these Type I derelicts never exceed two standard deviations from the mean at any point during the 60 -year prediction period. Thus, from the perspective of longitude/latitude space, these derelicts are "strongly" synchronized with the latitudinal motion in Figure 1 (i.e., the conga-line), and maintain this synchronization over the full 53-year cycle.

Conversely, Figure 6(b) provides predicted RAAN trajectories for the eight unsynchronized objects in Table 2, which have initial RAAN angles greater than two standard deviations away from the distribution mean. Continuous nodal regression of these Type II trajectories drives the RAAN angles of these outliers - and thus their daily latitude cycles - from being unsynchronized on 01 March 2014 into strong synchronization in an estimated 25-35 years. After approaching and achieving the distribution mean, however, the RAAN angles for these Type II derelicts continue to regress, until these objects once again become unsynchronized in 45-55 years, during which the node rapidly regresses as the minimum inclination of the cycle is achieved. These "patterns of life" for Type II objects thus differ from those of Type I objects in that although Type I trajectories admit synchronized latitude cycles over the entirety of the 53-year progression, Type II trajectories result in latitudinal motion that shifts from unsynchronized to synchronized - and back - during a single progression. The apparent anomalous latitude motion observed in Figure 1 can therefore be explained by the Type II progressions that these eight derelicts are traversing in inclination/RAAN space. Since natural transitions from a Type I to Type II cycle (or vice versa) will not occur under conservative gravitational perturbations alone, the three possibilities noted in Section 3 apply if assessing how or why these objects are following Type II cycles.

\section{Conclusions}

In this paper, the wave-like synchronization exhibited by the derelict population at GEO - the conga-line motion - is explained using bottom-up, analytical theory that describes inclination and RAAN variations driven by 


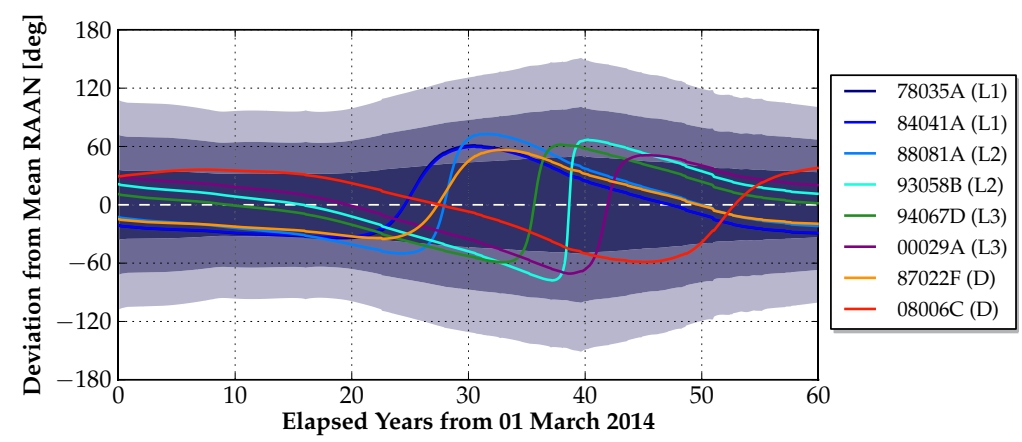

(a) Projected 60-year RAAN profiles for initially synchronized GEO derelicts.

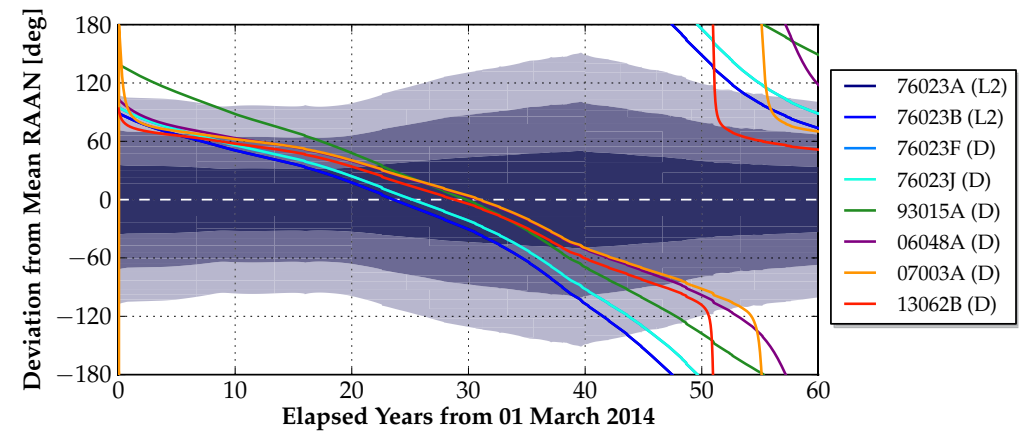

(b) Projected 60-year RAAN profiles for initially unsynchronized GEO derelicts.

Figure 6: RAAN histories for initially synchronized and initially unsynchronized objects near GEO on 28 February 2014, predicted using the doubly-averaged Equations (1)-(2). Shaded swaths indicate $\pm 1, \pm 2$, and \pm 3 standard deviations from the distribution mean, which expand and contract over the course of the 53-year cycle, reflecting the future distributions illustrated in Figure 5.

luni-solar perturbations and Earth's oblateness over a 53-year period. This well-known theory is combined with top-down, operational observation data to paint a portrait of the current inclination and RAAN distribution by controlled or uncontrolled class and age on-orbit, and how this distribution will evolve under the approximate doubly-averaged Equations (1)-(2). Lastly, derelicts with partially or fully out-of-phase latitude cycles are explained not by apparent anomalous motion, but rather in terms of the difference between Type I and Type II progressions in the phase space. 
Several operational considerations arising from the synchronized debris motion at GEO are highlighted in this paper with applications to flight safety, anomaly correlation, and space situational awareness activities in this regime. Naturally-occurring Type II motion - which arises from the combination of inclination, RAAN, and semi-major axis when the satellite is abandonedexplains not only why younger objects lag behind older objects in the daily latitude cycle, but also shows how the latitude cycle of a Type II object shifts into and out of synchronization with the Type I population over the course of the 53-year cycle. For sensor tasking, predictions of the combined Type I/II motion over a projected 60-year time frame highlight that the GEO pinch points resulting from tight clustering in RAAN are beginning to spread out, achieving maximum dispersion in about 40 years. As a result, both groundand space-based surveillance systems will require an increasingly wider search fence in right ascension to maintain demanded levels of sensor productivity in the near future.

\section{Acknowledgments}

The authors would like to acknowledge the U.S. Department of Defense and the National Defense Science and Engineering Graduate Fellowship (NDSEG), the program through which funding for this research was obtained. The authors would also like to thank the anonymous reviewers who strengthened the content of this manuscript.

\section{References}

Darren S. McKnight and Frank R. Di Pentino. New Insights on the Orbital Debris Collision Hazard at GEO. Acta Astronautica, 85:73-82, 2013.

K. S. Capelle and J. Sharma. Geosynchronous Satellite Orbit Pattern: Improvements to SBV Geosynchronous Search. In Proceedings of the 2000 Space Control Conference, pages 29-42. MIT Lincoln Laboratory, April 2000 .

T. Flohrer. Classification of Geosynchronous Objects: Issue 16. Technical Report 1, European Space Operations Centre, February 2014.

Chia-Chun Chao. Applied Orbit Perturbation and Maintenance. The Aerospace Press, 2005. 
Vladimir A. Chobotov. Orbital Mechanics. American Institute of Aeronautics and Astronautics, Inc., 2002.

David Vallado. Fundamentals of Astrodynamics and Applications. Microcosm Press, 3 edition, 2007.

R. R. Allan and G. E. Cook. The Long-Period Motion of the Plane of a Distant Circular Orbit. Proceedings of the Royal Society of London, Series A, Mathematical and Physical Sciences, 280(1380):97-109, July 1964.

Larry Jay Friesen, Donald J. Kessler, and Herbert A. Zook. Reduced Debris Hazard Resulting from a Stable Inclined Geosynchronous Orbit. Advances in Space Research, 13(8):231-241, 1993.

Chang-Yin Zhao, Ming-Jiang Zhang, Hong-Bo Wang, Jian-Ning Xiong, TingLei Zhu, and Wei Zhang. Analysis on the Long-Term Dynamical Evolution of the Inclined Geosynchronous Orbits in the Chinese BeiDou Navigation System. Advances in Space Research, 56(3):377-387, August 2015.

S. H. Vaughan and T. L. Mullikin. Long Term Behavior of Inactive Satellites and Debris near Geosynchronous Orbits. In Proceedings of the 1995 AAS/AIAA Spaceflight Mechanics Conference, volume 89, pages 1571$1585,1995$.

Darren S. McKnight, Frank R. Di Pentino, and Patrick Dingman. SemiEmpirical Satellite Anomalies Analysis Highlighting Contributions from the Fengyun-1C. In Proceedings of the 64th International Astronautical Congress, 2013.

T. Yasaka, T. Hanada, and H. Hirayama. GEO Debris Environment: A Model to Forecast the Next 100 Years. Advances in Space Research, 23(1): 191-199, 1999.

Paul V. Anderson and Hanspeter Schaub. Local Orbital Debris Flux Study in the Geostationary Ring. Advances in Space Research, 51(12):2195-2206, June 2013.

Jayant Sharma, Grant H. Stokes, Curt von Braun, George Zollinger, and Andrew J. Wiseman. Toward Operational Space-Based Space Surveillance. Lincoln Laboratory Journal, 13(2):309-334, 2002. 
R. R. Allan. Perturbations of a Geostationary Satellite by the LongitudeDependent Terms in the Earth's Gravitational Field. Planetary and Space Science, 11:1325-1334, August 1963.

Chang-Yin Zhao, Ming-Jiang Zhang, Hong-Bo Wang, Wei Zhang, and JianNing Xiong. Two-Dimensional Phase Plane Structure and the Stability of the Orbital Motion for Space Debris in the Geosynchronous Ring. Advances in Space Research, 52(4):677-684, August 2013.

Hanspeter Schaub and John L. Junkins. Analytical Mechanics of Space Systems. American Institute of Aeronautics and Astronautics, Inc., 2nd edition, 2009.

T. Schildknecht. Optical Surveys for Space Debris. Astronomy and Astrophysics Review, 14(1):41-111, 2007.

T. Flohrer, T. Schildknecht, R. Musci, and E. Stoveken. Performance Estimation for GEO Space Surveillance. Advances in Space Research, 35(7): 1226-1235, 2005.

T. Flohrer, H. Krag, H. Klinkrad, and T. Schildknecht. Feasibility of Performing Space Surveillance Tasks with a Proposed Space-Based Optical Architecture. Advances in Space Research, 47(6):1029-1042, 2011. 